\title{
Modulation of Opiate-Related Signaling Molecules in Morphine-Dependent Conditioned Behavior: Conditioned Place Preference to Morphine Induces CREB Phosphorylation
}

\author{
José A Morón ${ }^{1,2,3}$, Srinivas Gullapalli',2, Chirisse Taylor', Achla Gupta', Ivone Gomes' and Lakshmi A Devi*,', \\ 'Department of Pharmacology and Systems Therapeutics, Mount Sinai School of Medicine, New York, NY, USA
}

\begin{abstract}
Opiate addiction is a chronic, relapsing behavioral disorder where learned associations that develop between the abused opiate and the environment in which it is consumed are brought about through Pavlovian (classical) conditioning processes. However, the signaling mechanisms/pathways regulating the mechanisms that underlie the responses to opiate-associated cues or the development of sensitization as a consequence of repeated context-independent administration of opiates are unknown. In this study we examined the phosphorylation levels of various classic signaling molecules in brain regions implicated in addictive behaviors after acute and repeated morphine administration. An unbiased place conditioning protocol was used to examine changes in phosphorylation that are associated with $(I)$ the expression of the rewarding effects of morphine and (2) the sensitization that develops to this effect. We also examined the effects of a $\delta$-receptor antagonist on morphine-induced conditioned behavior and on the phosphorylation of classic signaling molecules in view of data showing that blockade of $\delta$-opioid receptor $(\delta \mathrm{OR})$ prevents the development of sensitization to the rewarding effects of morphine. We find that CREB phosphorylation is specifically induced upon the expression of a sensitized response to morphine-induced conditioned behavior in brain areas related to memory consolidation, such as the hippocampus and cortex. A similar effect is also observed, albeit to a lesser extent, in the case of the GluRI subunit of AMPA glutamate receptor. These increases in the phosphorylation levels of CREB and PGluRI are significantly blocked by pretreatment with a $\delta \mathrm{OR}$ antagonist. These results indicate a critical role for phospho-CREB, AMPA, and $\delta$ OR activities in mediating the expression of a sensitized response to morphine-dependent conditioned behavior.
\end{abstract}

Neuropsychopharmacology (2010) 35, 955-966; doi:I0.1038/npp.2009.199; published online 2 December 2009

Keywords: addiction; morphine; opioid receptors; sensitization; drug reward

\section{INTRODUCTION}

Opiate addiction is a complex relapsing brain disease process that is characterized by the compulsive seeking and taking of an opiate, despite adverse consequences, and the emergence of a negative emotional state when access to the opiate is denied (Koob et al, 1998). Relapse in abstinent human opiate addicts can be triggered by a number of events, including exposure to environmental cues associated with drug use (O'Brien et al, 1992). The learned associations that develop between the abused opiate and the

*Correspondence: Dr LA Devi, Department of Pharmacology and Systems Therapeutics, Mount Sinai School of Medicine, One Gustave L Levy Place, New York, NY 10029, USA,

Tel: + I 212241 8345, Fax: + I 2129967214 ,

E-mail: lakshmi.devi@mssm.edu

${ }^{2}$ These authors contributed equally to this work.

${ }^{3}$ Current address: Department of Pharmacology and Toxicology, Center for Addiction Research, University of Texas Medical Branch, Galveston, TX, USA.

Received 17 June 2009; revised 28 October 2009; accepted 29 October 2009 environment in which it is consumed are brought about through Pavlovian (classical) conditioning processes: repeated use of the drug in a particular environment generates associations between the drug and the environment, such that subsequent exposure to the environment in the absence of the drug elicits physiological and behavioral responses similar to those induced by the drug itself (O'Brien et al, 1992). These effects are long lasting and can occur despite years of abstinence from drug use (O'Brien et al, 1992). However, little is known about the molecular mechanisms that underlie drug-dependent learned associations.

The conditioned place preference (CPP) paradigm is an animal model widely used to investigate the mechanisms underlying context-dependent learning associated with drugs of abuse (Bardo et al, 1995). Analogous to the drugenvironment associations that trigger relapse in humans (O'Brien et al, 1992) the CPP paradigm is based on Pavlovian conditioning, where the abused drug (unconditioned stimulus) is repeatedly paired with a specific environment that becomes a conditioned stimulus (CS). Subsequently, when animals have the choice of exploring the previously drug-paired $(C S+)$ environment and a 
previously nondrug-paired environment, they prefer the CS + environment (conditioned response) (Bardo et al, 1995). Thus, this model can be used to explore the molecular mechanisms that govern expression of conditioned responses to drug-associated cues. Several reports have shown that the conditioned rewarding effects of $\mu$-opiod receptor $(\mu \mathrm{OR})$ agonists are enhanced in animals with a history of opiate exposure (Shippenberg et al, 1996, 1998). Indeed, the efficacy of morphine to elicit a CPP is enhanced when the number of days of environment-drug pairing are increased before the conditioning procedure (Shippenberg et al, 1996).

In general, the field of addiction research has focused primarily on the mesolimbic dopamine pathway, which originates in the ventral tegmental area (VTA) and projects to the nucleus accumbens (Nac) and prefrontal cortex (PFC), because of its critical role in mediating the rewarding actions of virtually all drugs of abuse (Everitt and Wolf, 2002). However, the importance of learning and memory in the process of addiction is becoming increasingly clear. Learning and memory are primary components for the development of context-associated cues. Indeed, recent evidence underscores a prominent role for limbic brain areas (like the hippocampus and cortex) in the control of behaviors reinforced by natural rewards (eg, food), as well as psychoactive drugs (Fuchs et al, 2005; Nestler, 2001). For example, the hippocampus has a key role in associative memory networks, the encoding and consolidation of novel environmental information, and in the learning of relational information between environmental stimuli (Morris et al, 2003). In addition, the hippocampus has been implicated in the regulation of morphine-dependent conditioned behavior (Corrigall and Linseman, 1988; Ferbinteanu and McDonald, 2001). Thus, both the hippocampus and cortex are likely to be important in the expression of drugdependent conditioned behavior.

A number of studies showed that opiate addiction involves cellular mechanisms historically associated with memory formation (Hyman and Malenka, 2001) such as the stimulation of serine/threonine kinases such as mitogenactivated protein kinase (MAPK) and calcium/calmodulindependent kinase (CAMK) II (Fukuda et al, 1996; Polakiewicz et al, 1998; Law et al, 2000). In addition, the transcription factor CREB is widely implicated in many forms of neuronal plasticity, including drug addiction (Carlezon et al, 2005; Nestler, 1997). Thus, changes in CREB activity may be important in mediating mechanisms underlying the expression of a conditioned response to an opiate-paired environment. Also, accumulating evidence indicates that opiates can modulate synaptic transmission and plasticity by altering glutamatergic transmission and long-term potentiation in brain areas such as the hippocampus and cortex ( $\mathrm{Pu}$ et al, 2002; Xu et al, 2003). This suggests that complex adaptive mechanisms take place during repeated exposure to opiates. A better understanding of the molecular mechanisms involved in drug-associated conditioned responses will be extremely useful in the development of novel pharmacological tools for intervention so as to prevent development of opiate addiction.

Several studies indicate that the $\delta$-opioid receptor $(\delta \mathrm{OR})$ may have a substantial role in the regulation of morphine-induced rewarding properties (see review by Narita et al, 2001). Indeed, a recent study shows that selective blockade of $\delta \mathrm{OR}$ prevents sensitization to the conditioning rewarding effects of morphine (Shippenberg et al, 2009). This effect is likely to be due to a functional interaction between $\mu \mathrm{OR}$ and $\delta \mathrm{OR}$ (Gomes et al, 2004). This is in agreement with a recent report that shows that a physical interaction between $\mu \mathrm{OR}$ and $\delta \mathrm{OR}$ modulates $\mu \mathrm{OR}$ mediated dependence (Daniels et al, 2005).

In this study we examined changes in the extent of phosphorylation of various signaling molecules (such as MAPK, CaMKII, CREB, and GluR1) under different paradigms of morphine administration. We find that acute morphine treatment (single injection) leads to significant decreases in CREB phosphorylation in all brain regions studied. Interestingly, on context-dependent administration of morphine (morphine CPP) CREB phosphorylation is increased in hippocampus and cortex with a parallel increase in the phosphorylation of the GluR1 subunit of the AMPA receptor. These results are also observed on a paradigm of sensitization to morphine CPP. In contrast, repeated context-independent exposure to morphine, following a paradigm which is comparable to the one that elicits sensitization to morphine CPP, leads to a decrease in CREB phosphorylation in the hippocampus and cortex. Next, we examined whether $\delta$ OR blockade, which attenuates sensitization of morphine CPP but not the conditioning or reward, alters the phosphorylation state of these molecules. Results obtained indicate that blockade of sensitization to morphine CPP with a $\delta \mathrm{OR}$ antagonist correlates with a decrease in the phosphorylation state of these molecules in the hippocampus and cortex. These data suggest that $\delta \mathrm{OR}$ is recruited after repeated morphine administration and that its activity is critical for sensitization to a conditioned response to morphine.

\section{MATERIALS AND METHODS}

\section{Animals}

Male Sprague-Dawley rats (200-250 g; Harlan) were used in all studies. Rats were group-housed in accordance with NIH guidelines on a 12-h light-dark cycle with food and water available ad libitum. All experimental protocols were approved by the Mount Sinai School of Medicine Institutional Animal Care and Use Committee.

\section{Drugs}

Morphine sulfate and naltriben were purchased from Sigma (St Louis, MO). Morphine or naltriben were dissolved in sterile saline and administered subcutaneously (s.c.). All vehicle injections consisted of sterile saline.

\section{Single Drug Treatment}

Animals were administered a single dose of morphine $(10 \mathrm{mg} / \mathrm{kg}$, s.c.) or saline and killed either $30 \mathrm{~min}$ (acute $30 \mathrm{~min}$ ) or $48 \mathrm{~h}$ (acute $48 \mathrm{~h}$ ) after morphine administration. Brains were rapidly extracted, different brain regions were dissected out and frozen at $-80^{\circ} \mathrm{C}$ till use. 


\section{Conditioned Place Preference}

The CPP chambers are housed within sound- and lightattenuating cabinets and contained three distinct sensory environments. The conditioning chambers are differentiated on the basis of wall color and flooring, with the white conditioning chamber containing a steel mesh floor, the black conditioning chamber containing a floor made of steel rods, and the center gray chamber containing a sheet-metal floor. Guillotine doors connect each side compartment to the center compartment. Rats were conditioned using a modified standard place conditioning procedure (Mueller et al, 2002). The CPP procedure consisted of three distinct phases: preconditioning, conditioning, and test. In the preconditioning phase (day 1), rats were placed in the gray center compartment and allowed to freely roam the entire apparatus for $30 \mathrm{~min}$. The amount of time spent in each compartment (black or white) was recorded to assess the unconditioned preference and then animals were returned to their home cages. Any rat showing a strong unconditioned preference $(>9 \mathrm{~min})$ was excluded. On the subsequent day, the conditioning phase (days 2-7) was initiated. The conditioning sessions were conducted twice each day with a $6 \mathrm{~h}$ intervening interval. During conditioning sessions, the animals received a s.c. treatment injection and were confined for $30 \mathrm{~min}$ to the assigned compartment. After $6 \mathrm{~h}$ animals were administered the second conditioning treatment and confined to the other compartment for $30 \mathrm{~min}$. Rats were divided into two treatment groups. The morphine-paired group received morphine $(10 \mathrm{mg} / \mathrm{kg})$ in one side of the CPP chamber (morphine-paired) and saline in the other side (nondrug-paired) of the chamber. The treatment compartment was assigned according to a counterbalanced design, such that one half of the animals in each experimental group were conditioned with morphine to the spontaneously preferred side and the other half to the spontaneously nonpreferred side. The order of presentation of morphine and saline was counterbalanced. The saline control group received saline in both sides of the CPP chamber. On the test day (day 8), animals were placed on the central gray chamber and allowed to freely roam the entire apparatus. Time spent in each test compartment was recorded separately for each animal for $10 \mathrm{~min}$. The change of preference was calculated as the difference (in seconds) between the time spent in the drug-paired compartment on the testing day and the time spent in this compartment in the preconditioning session. Animals were killed immediately after behavioral testing and their brains were rapidly extracted and different brain regions dissected out and frozen at $-80^{\circ} \mathrm{C}$ till use.

\section{Sensitization to Morphine CPP}

Conditioning was also performed following a morphine sensitization paradigm developed by Shippenberg et al (2009) in which animals that received prior repeated injections of the drug show robust place preference even at doses that are ineffective in producing CPP in naive animals. Preconditioning was performed as described above. After that, animals received once-daily injections of saline or morphine $(5 \mathrm{mg} / \mathrm{kg}$ ) for 5 days in their home cages. On days 6 and 7 animals did not receive any treatment, because it has been described that a period of abstinence is necessary for the expression of this sensitized response to morphine CPP (Shippenberg et al, 1996).

Place conditioning in the CPP chambers was carried out on days 8 and 9 following a counterbalanced design as described above. On each of these days, animals received one conditioning session with saline and one with morphine $(5 \mathrm{mg} / \mathrm{kg})$. A total of four conditioning sessions were carried out as previous studies in our laboratory have shown that four conditioning sessions are ineffective in producing a conditioned response in naive animals (Taylor and Devi, unpublished data). Animals were tested on the 10th day for time spent in the previously morphine-paired side. The change in preference was calculated as described above. Animals were killed immediately after behavioral testing and their brains were rapidly extracted and different brain regions dissected out and frozen at $-80^{\circ} \mathrm{C}$ till use.

To study the role of a $\delta \mathrm{OR}$ antagonist on sensitization to morphine CPP, we treated the animals with naltriben before morphine administration as described (Shippenberg et al, 2009) with some modifications. Briefly, animals received once-daily injections of naltriben $(0.1 \mathrm{mg} / \mathrm{kg})$ or vehicle in their home cages for 5 days. After $15 \mathrm{~min}$, they received an injection of morphine $(5 \mathrm{mg} / \mathrm{kg})$. Conditioning sessions commenced on day 8 as described above. During conditioning animals received once-daily injection of naltriben $(0.1 \mathrm{mg} / \mathrm{kg}) 15 \mathrm{~min}$ before the first saline or morphine injection. Animals were tested on the 10th day, the change in the preference was calculated, and tissue was collected and kept as described above.

\section{Repeated Morphine Administration}

This paradigm of repeated morphine administration (without CPP) was designed to be comparable to the paradigm of sensitization to morphine CPP described above. Briefly, rats received once-daily injections of saline or morphine $(5 \mathrm{mg} / \mathrm{kg})$ in their home cages for 5 days. On days 6 and 7, animals did not receive any treatment. On days 8 and 9, animals received once-daily injections of morphine $(5 \mathrm{mg} / \mathrm{kg})$ or saline again in their home cages. On the 10th day, rats were killed and their brains were rapidly extracted and different brain regions dissected out and frozen at $-80^{\circ} \mathrm{C}$ till use.

\section{Western Blot Analysis}

Protein extracts from the different brain regions were obtained by homogenization in $62.5 \mathrm{mM}$ Tris ( $\mathrm{pH} 6.8$ ), $1 \%$ SDS containing a protease and phosphatase inhibitor cocktail (Sigma). Total protein concentrations were determined using BCA protein assay reagent (Pierce). Equal amounts of total protein were resolved on 10\% SDS-PAGE gels and transferred to nitrocellulose membranes (Schleicher and Schuell BioScience) by electroblotting. Membranes were blocked with $5 \%$ dry milk and then incubated with either total p44/42 MAPK, phospho-p44/42 MAPK (Thr202/ Tyr204) (1:1000; Cell Signaling), total CaMKII $\alpha$ (1:10 000) phospho-CaMKII $\alpha$ (Thr286) (1:1000; Upstate), total GluR1, phospho-GluR1 (Ser831) (1:1000; Upstate), phospho-CREB (Ser133) (1:1000; Upstate) or actin (1:10000; Sigma, in case of phospho-CREB) antibodies to determine the extent of phosphorylation and to ensure equal protein loading and 
transfer. After incubation with secondary antibody $(1: 1000$ to $1: 2000$ anti-rabbit, Amersham Biosciences; $1: 2000$ to 1:20000 anti-mouse, Vector Laboratories) and extensive washing, membranes were incubated with ECL detection reagents (Pierce) and exposed to ECL Hyperfilm (Amersham Biosciences). Band densities were determined using NIH ImageJ software. Quantification was performed by measuring the intensity of the band with protein-specific antibodies.

\section{Data Analysis}

Results are expressed as the mean \pm SEM. Statistical comparisons were performed using one-way ANOVA with two-tailed unpaired $t$-test or with Tukey's test for multiple comparisons, where appropriate. Statistical analyses of data were carried out using GraphPad Prism, version 5.0.

\section{RESULTS}

To investigate the involvement of key signaling molecules in the neurochemical adaptations associated with the expression of morphine-dependent conditioned behavior, we first examined the effect of a single morphine injection $(10 \mathrm{mg} /$ $\mathrm{kg}$, s.c.) on the phosphorylation levels of p44/42 MAPK, CaMKII, GluR1, and CREB in different brain regions.

A comparison between saline- and morphine-treated animals $30 \mathrm{~min}$ after a single drug injection revealed significant decreases in the levels of pCREB in the Nac, VTA, striatum, PFC, hippocampus, and cortex in morphine- treated animals (Figure 1d). There were no significant changes in the levels of phosphorylated p44/42 MAPK, CaMKII, or GluR1 in any of the brain regions examined (Figure 1). Interestingly, $48 \mathrm{~h}$ after a single morphine injection there were significant increases in phosphorylated p44/42 MAPK and decreases in PCREB levels in VTA and hippocampus of morphine-treated animals (Figure 2). There were also significant decreases in PCREB levels in the striatum after morphine treatment (Figure 2). Thus, 2 days after an acute administration of morphine, we find differential alterations in the levels of phosphorylated signaling molecules in different regions of the brain.

Next, we examined the phosphorylation state of these proteins in different brain areas following morphine CPP. It is known that a certain number (that ranges from four to six depending on the dose of morphine) of conditioning sessions are necessary for the conditioning of morphine reward in rats that are opiate naive before place conditioning. In this study 6 days of conditioning led to a significant place preference to the drug as compared to saline controls (Figure 3a). Under this paradigm we find significant modulation of the levels of phosphorylated p44/42 MAPK, CaMKII, GluR1, and CREB in different brain regions. Levels of phosphorylated p44/42 MAPK significantly increased in the NAc, VTA, striatum, and hippocampus whereas the levels of pCREB were increased in all brain regions examined (Figure $3 \mathrm{~b}$ and e). There were also significant increases in the levels of phosphorylated CaMKII in the majority of regions examined (except VTA) in morphine-conditioned a
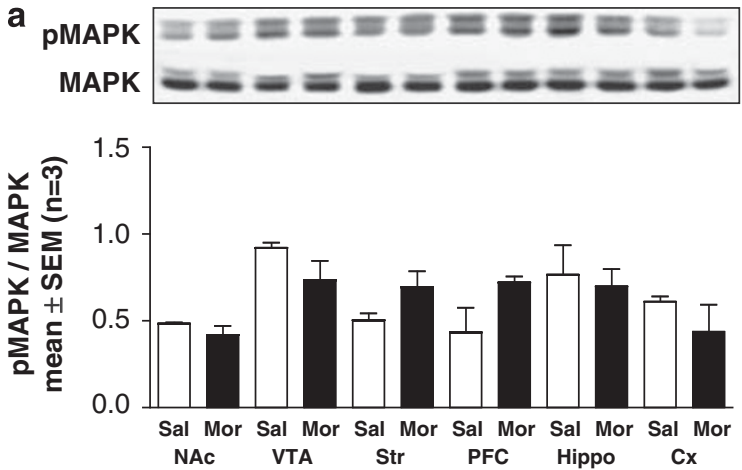

C
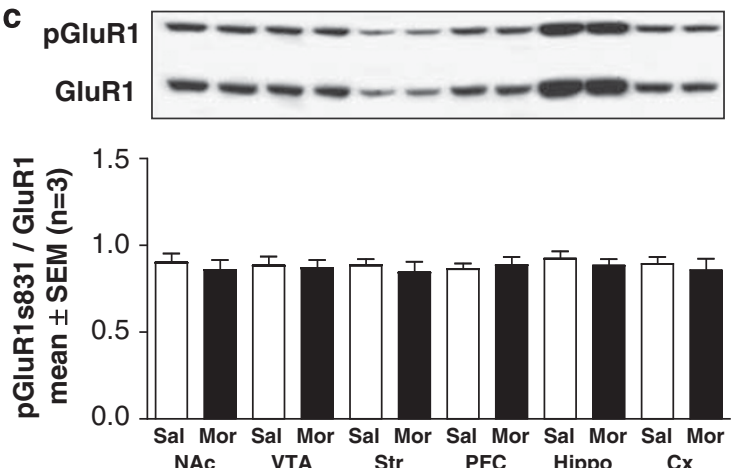

$42 / 44$ kd

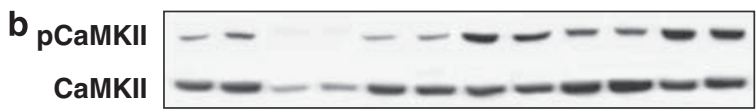

50 kd
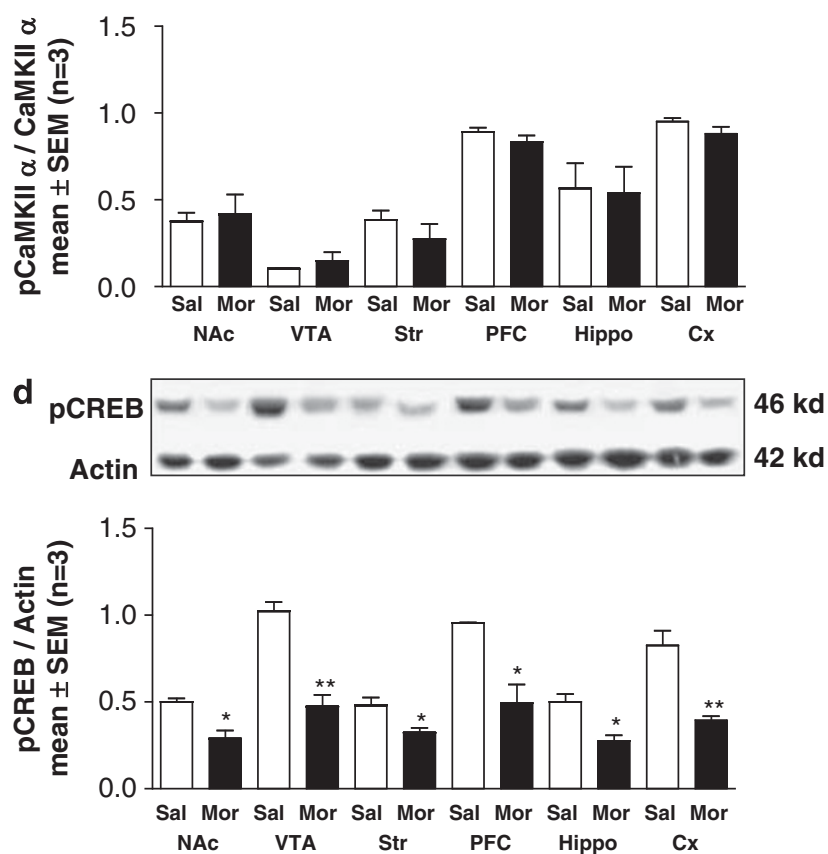

Figure I Modulation of mitogen-activated protein kinase (MAPK), calcium/calmodulin-dependent kinase II (CaMKII), GluRI, and CREB 30 min after acute morphine administration. Rats were treated with a single injection of saline or morphine ( $10 \mathrm{mg} / \mathrm{kg}$, s.c.) and killed 30 min later. Brain regions were dissected out and subjected to western blotting analysis using antibodies against pMAPK, total MAPK (a), pCaMKII, total CaMKII (b), pGluRI, total GluRI (c), pCREB, and actin (d) as described in the 'Materials and methods' section. A representative western blot is shown for each of the proteins studied. Data from multiple experiments (4-6 animals per group) were tabulated and presented as relative ratio of phosphorylated/total forms (mean \pm SEM). One-way ANOVA with unpaired two-tailed t-test, $n=4-6, * p<0.05 ; * * 0<0.01$. 
a
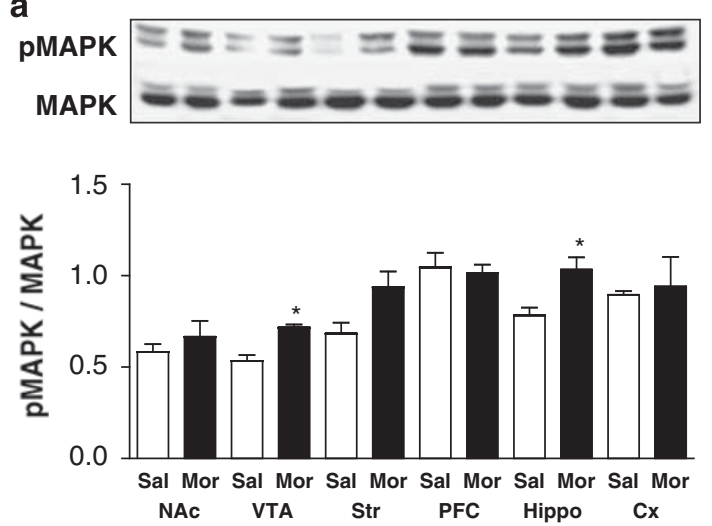

C
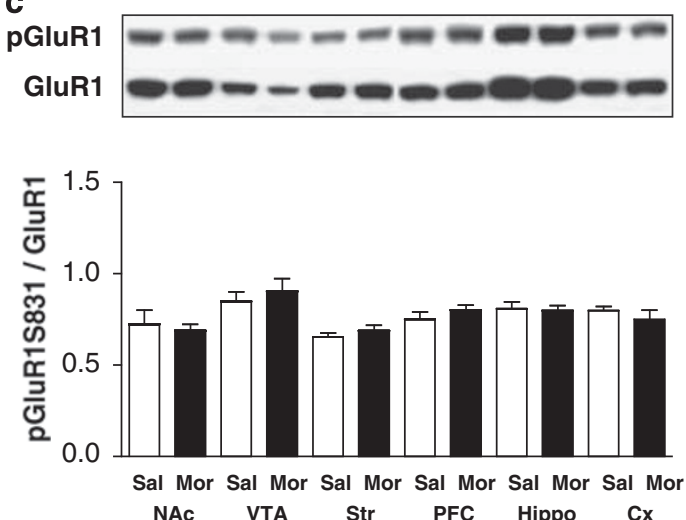

$42 / 44 \mathrm{kd}$

b
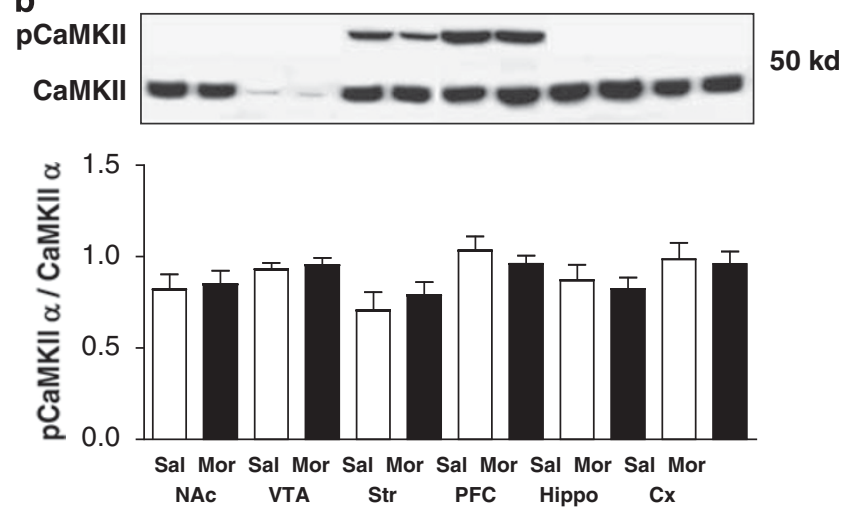

d

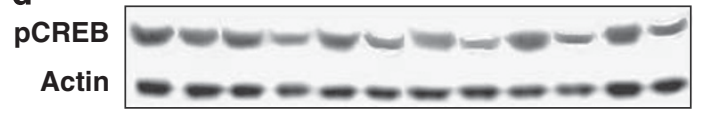

46 kd $108 \mathrm{kd}$

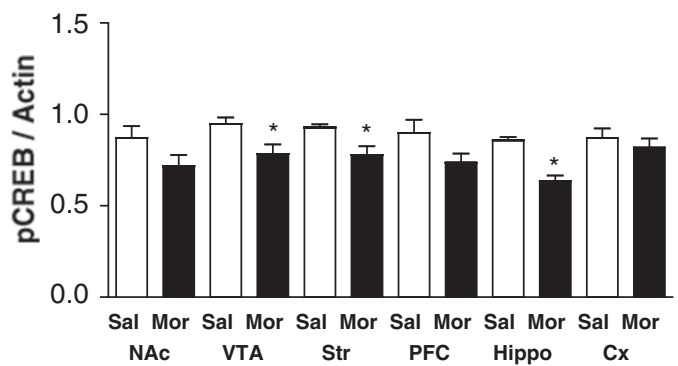

Figure 2 Modulation of mitogen-activated protein kinase (MAPK), calcium/calmodulin-dependent kinase II (CaMKII), GluRI, and CREB 48h after acute morphine administration. Rats treated with a single injection of saline or morphine ( $10 \mathrm{mg} / \mathrm{kg}$, s.c.) were killed $48 \mathrm{~h}$ later and the brain regions were subjected to western blotting analysis using antibodies against pMAPK, total MAPK (a), pCaMKII, total CaMKII (b), pGluRI, total GluRI (c), pCREB, and actin (d) as described in 'Materials and methods' section. A representative western blot is shown for each of the proteins studied. Data from multiple experiments (4-6 animals per group) were tabulated and presented as relative ratio of phosphorylated/total forms (mean \pm SEM). One-way ANOVA with unpaired two-tailed $t$-test, $n=4-6, * 0<0.05$.

animals (Figure 3c). Finally, there were increases in phospho GluR1 in NAc, hippocampus, and cortex (Figure 3d). Taken together, these results indicate that the expression of morphine-dependent conditioned responses induces differential alterations in the levels of signaling molecules in different brain regions.

Next, we evaluated the effects of a sensitized response to morphine CPP on the phosphorylation levels of p44/42 MAPK, CaMKII, GluR1, and CREB in different brain regions. It is known that in animals with a history of $\mu \mathrm{OR}$ agonist administration, the potency and efficacy of morphine in producing CPP are increased (Shippenberg et al, 1996, 1998). In addition, after the repeated administration of morphine and before the initiation of the conditioning sessions the animals do not receive any treatment for 2 days. This design is based on previous studies that indicate that a period of abstinence is necessary for the expression of the enhanced conditioned response (Shippenberg et al, 1996). Therefore, this paradigm of morphine administration mimics the state where intermittent administration of drugs of abuse leads to an enhancement of their behavioral effect (Kalivas and Stewart, 1991) that can last for several days after drug administration. This model therefore provides information on signaling mechanisms that underlie drug sensitization and may have a pivotal role in drug-dependent conditioned responses. Also this paradigm has been shown to induce enhanced place preference even at doses that are ineffective in producing CPP in naive animals (Shippenberg et al, 2009; Figure 4a). Under these conditions we find significant increases in the levels of phosphorylated p44/42 MAPK in NAc, PFC, and hippocampus (Figure $4 \mathrm{~b}$ ) and no changes in the levels of pCaMKII (Figure 4c). We also find increases in pGluR1 in striatum, PFC, and hippocampus (Figure 4d). In addition, there were increases in pCREB levels in all brain regions examined (except PFC; Figure 4e).

In addition, we examined whether these changes in the phosphorylation state of these molecules were specific to the expression of a sensitized response to morphine CPP or could also be due to repeated exposure to the drug. However, the above-mentioned protocol of sensitization to morphine CPP has some limitations. For example, the fact that during this protocol the animals do not receive any treatment for 2 days before the conditioning sessions are initiated could induce withdrawal, and this effect may be reflected in the data obtained. Therefore, to address both issues, during the repeated morphine administration paradigm the animals were treated at their home cages with the same doses of morphine and did not receive any treatment for 2 days. Repeated morphine administration did not result in any significant differences between saline- and morphine-treated animals with respect to phosphorylated p44/42 MAPK levels (Figure 5a). However, there were increases in the levels of pCaMKII in NAc, 

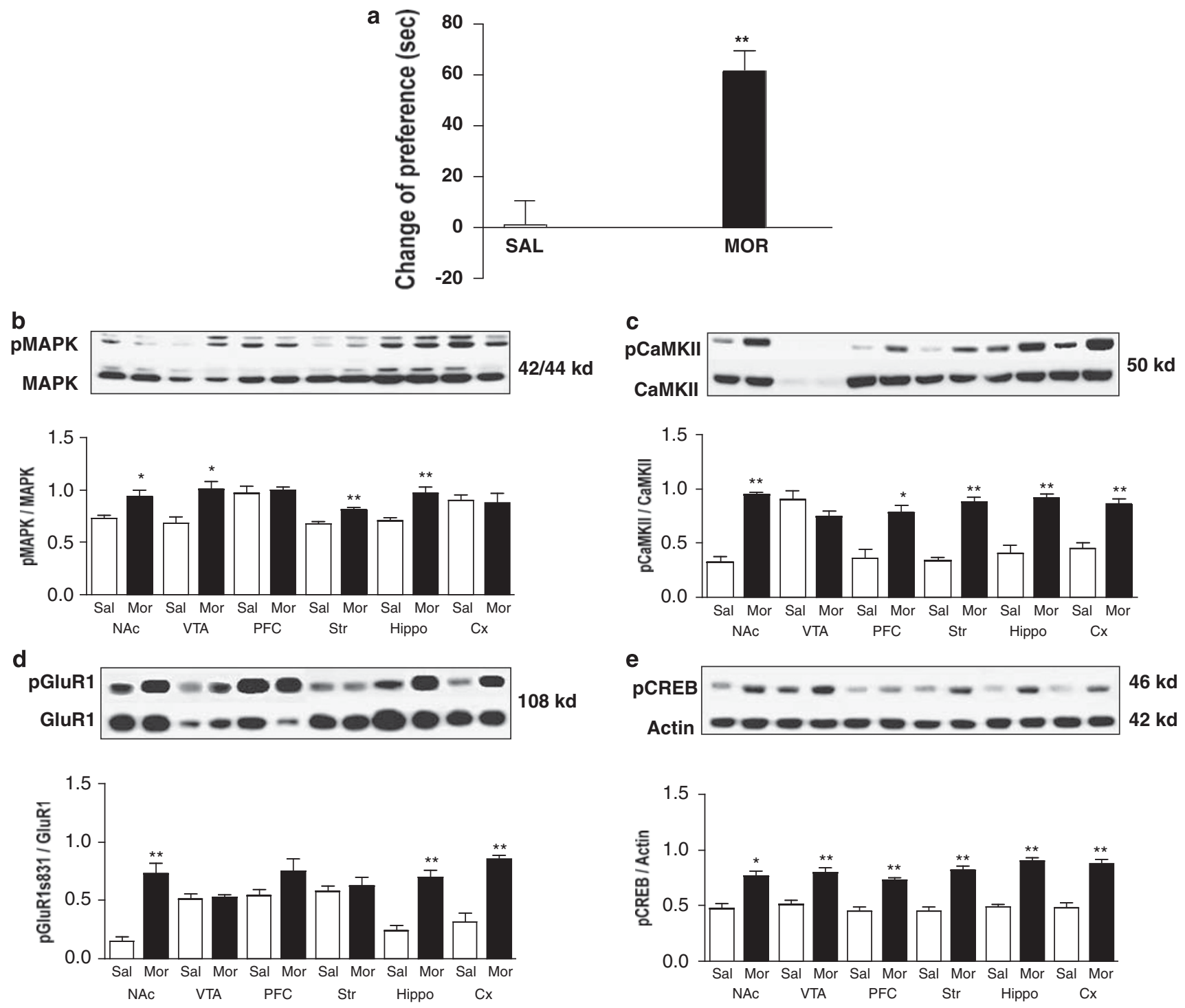

Figure 3 Conditioned place preference to morphine following a 6-day classical conditioning paradigm. (a) Rats (6-8 per group) were subjected to classical morphine conditioning paradigm as described in 'Materials and methods' section. Data represent the change of preference (the difference between the time spent in the drug-paired compartment on the testing day and the basal preference time spent before drug treatment) (mean \pm SEM). Rats showed a significant preference for the morphine-paired chamber (unpaired two-tailed $t$-test, $n=6-8, p<0.0 \mathrm{I}$ ). (b-e) Phosphorylation pattern of mitogen-activated protein kinase (MAPK), calcium/calmodulin-dependent kinase II (CaMKII), GluRI, and CREB upon classical morphine conditioning. The brains from rats were collected after measuring conditioned place preference (CPP) and various brain regions were subjected to western blotting analysis using antibodies against pMAPK, total MAPK (b), pCaMKII, total CaMKII (c), pGluRI, total GluRI (d), pCREB, and actin (e) as described in 'Materials and methods' section. A representative western blot is shown for each of the proteins studied. Data from multiple experiments (6-8 animals per group) were tabulated and presented as relative ratio of phosphorylated/total forms (mean \pm SEM). One-way ANOVA with unpaired two-tailed $t$-test, $n=6-8, * p<0.05, * * p<0.01$.

striatum, and PFC, whereas levels of pGluR1 were increased only in PFC (Figure $5 b$ and c). We also find significant decreases in PCREB levels only in hippocampus and cortex of morphine-treated animals with no changes observed in other brain regions (Figure 5d). These findings greatly contrast findings under conditions of morphine CPP (Figure 4), indicating differential alterations in the levels of signaling molecules in distinct brain regions that are modified on expression of morphine CPP or the sensitization that develops to this conditioned response (Figures 4 and 5). Taken together, these results indicate that there are substantial differences in phosphorylation of signaling molecules under different paradigms of morphine-dependent conditioned behavior.
A number of studies have shown that the rewarding effects of morphine can be modulated by either blocking $\delta \mathrm{OR}$ activity (by administration of $\delta \mathrm{OR}$ antagonists) or reducing the levels of $\delta \mathrm{OR}$ (as seen in knockout animals or by administration of antisense oligonucleotides) (Abdelhamid et al, 1991; Sanchez-Blazquez et al, 1997; Zhu et al, 1999). On the basis of previous findings (Shippenberg et al, 2009), we examined whether pretreatment with the $\delta$ OR receptor antagonist, naltriben, would modulate the sensitization that develops to morphine CPP. A dose of $0.1 \mathrm{mg} / \mathrm{kg}$ naltriben was selected to selectively block the $\delta$ OR 2 subtype (Sofouglu et al, 1991; Portoghese et al, 1992). We see that saline pretreatment had no significant effect in place preference whereas naltriben pretreatment completely 


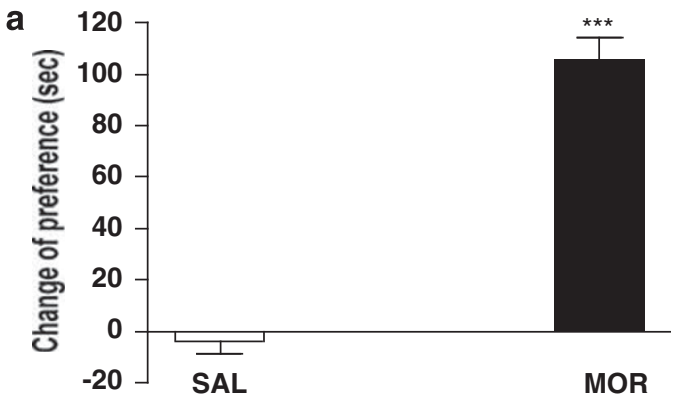

b
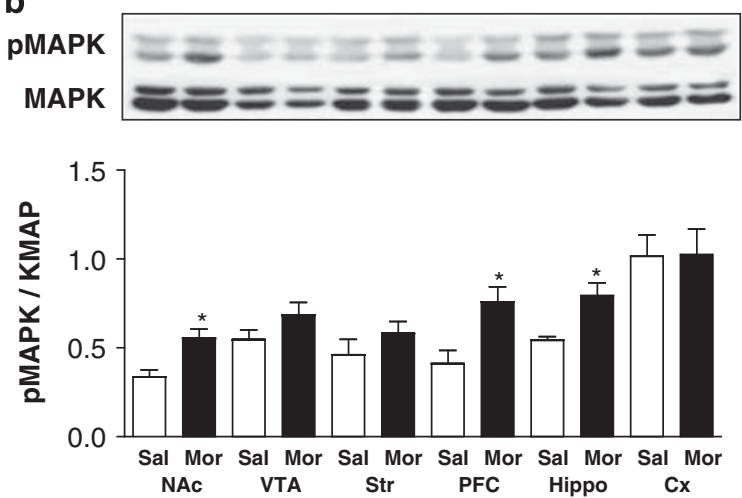

d

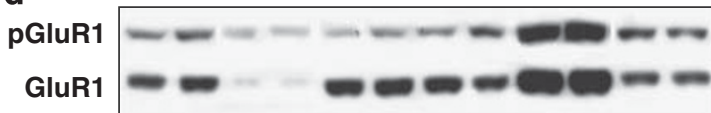

$108 \mathrm{kd}$

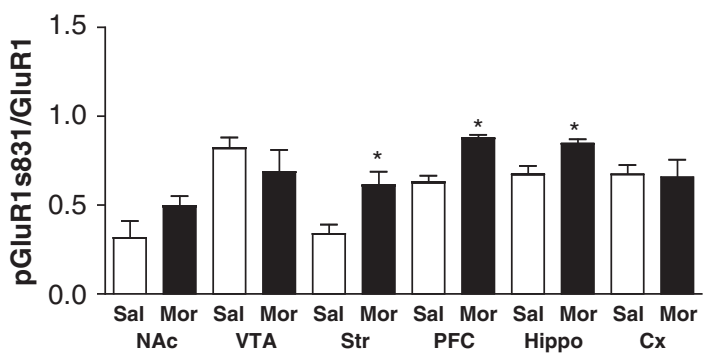

$42 / 44 \mathrm{kd}$
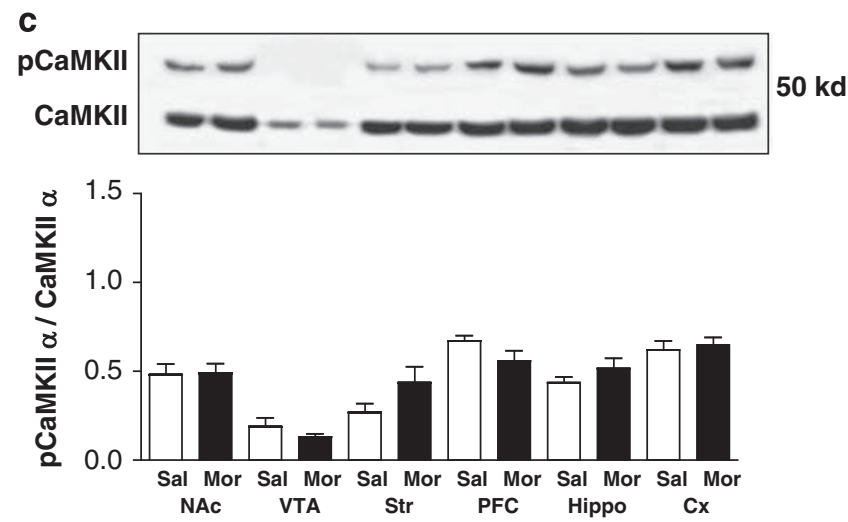

e
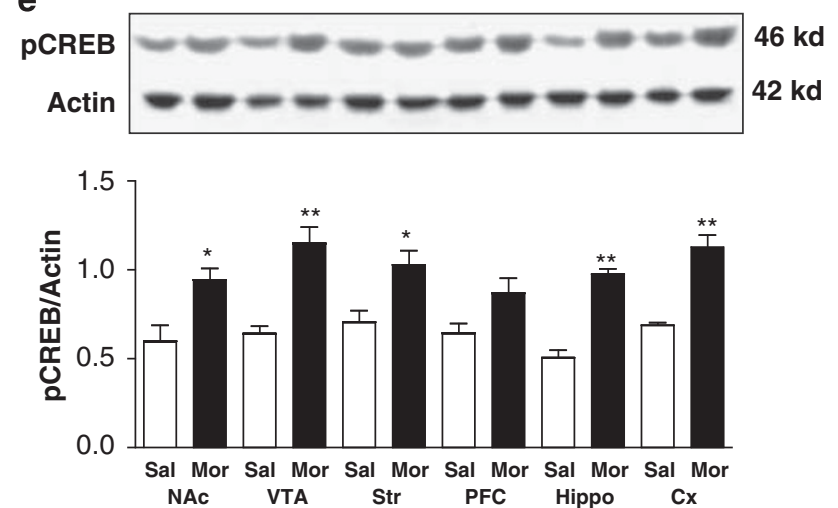

Figure 4 Conditioned place preference to morphine following a sensitization paradigm. (a) Rats (6-8 per group) were subjected to morphine sensitization and conditioning paradigm as described in 'Materials and methods' section. Data represent the change of preference (the difference between the time spent in the drug-paired compartment on the testing day and the basal preference time for this compartment before drug treatment) $($ mean $\pm \mathrm{SEM})$. Rats showed a stronger preference for the morphine-paired chamber (unpaired two-tailed $t$-test, $n=6-8, * * * p<0.0 \mathrm{I})$. (b-e) Phosphorylation pattern of mitogen-activated protein kinase (MAPK), calcium/calmodulin-dependent kinase II (CaMKII), GluRI, and CREB on morphine sensitization and conditioning. The brains from rats were collected after measuring conditioned place preference (CPP) and various brain regions were subjected to western blotting analysis using antibodies against pMAPK, total MAPK (b), pCaMKII, total CaMKII (c), pGluRI, total GluRI (d), pCREB, and actin (e) as described in 'Materials and methods' section. A representative western blot is shown for each of the proteins studied. Data from multiple experiments (6-8 animals per group) were tabulated and presented as relative ratio of phosphorylated/total forms (mean \pm SEM). One-way ANOVA with unpaired twotailed $t$-test, $n=6-8$, ** $p<0.01$.

blocked the sensitized response that develops to morphine CPP (Figure 6). Next, we examined the effect of pretreatment with naltriben on the levels of phosphorylation of CREB and GluR1, two of the molecules shown to be significantly phosphorylated during the morphine CPP sensitization paradigm (Figure 4). We find that the morphine CPPmediated increase in PCREB levels seen in hippocampus, cortex, and VTA was completely blocked when animals were pretreated with naltriben (NTB + Mor; Figure 7). In salinetreated controls, pretreatment with naltriben had no significant effect in the levels of pCREB (NTB + Sal; Figure 7). We also find that morphine CPP-mediated increase in
pGluR1 seen in hippocampus, cortex, PFC, and VTA was also completely blocked by pretreatment with naltriben (NTB + Mor; Figure 8). Pretreatment with naltriben in salinetreated animals led to increases in pGluR1 levels in VTA with no significant changes in other brains areas examined (Figure 8), suggesting a role for endogenous $\delta \mathrm{OR}$ activity in this brain region. Taken together these results indicate that pretreatment with the $\delta \mathrm{OR}$ antagonist, naltriben, blocks the sensitization that develops following morphine CPP as well as the related increases in PCREB and pGluR1 levels especially in hippocampus and cortex, brain regions implicated in memory consolidation. 
a

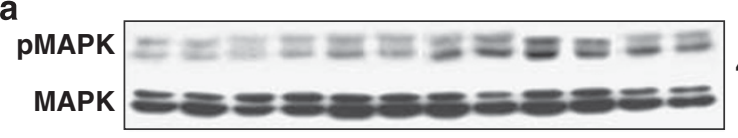
$42 / 44 \mathrm{kd}$

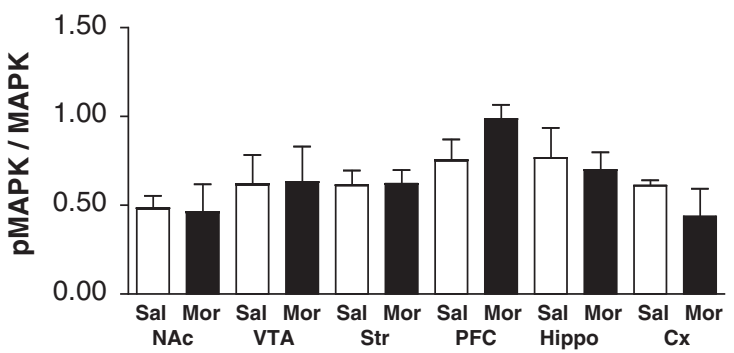

C
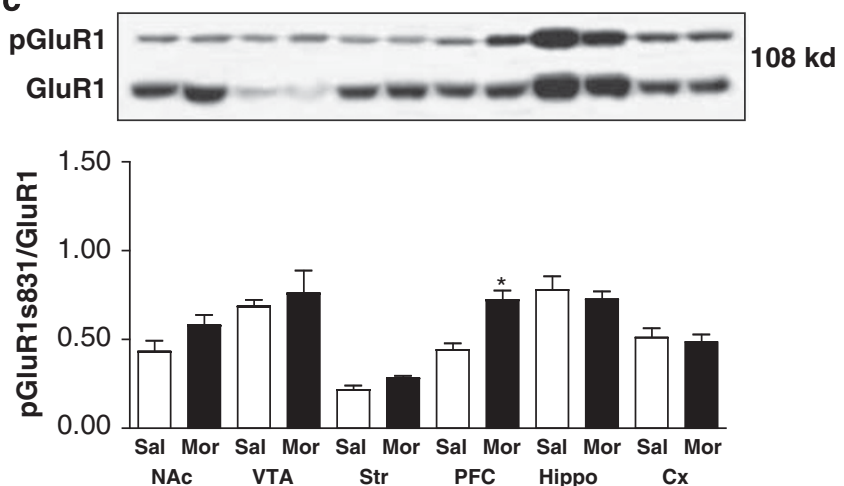

b

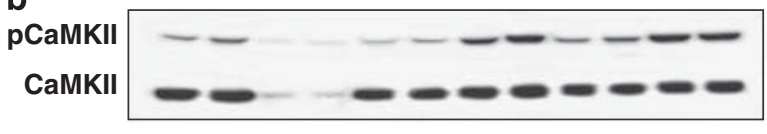

50 kd
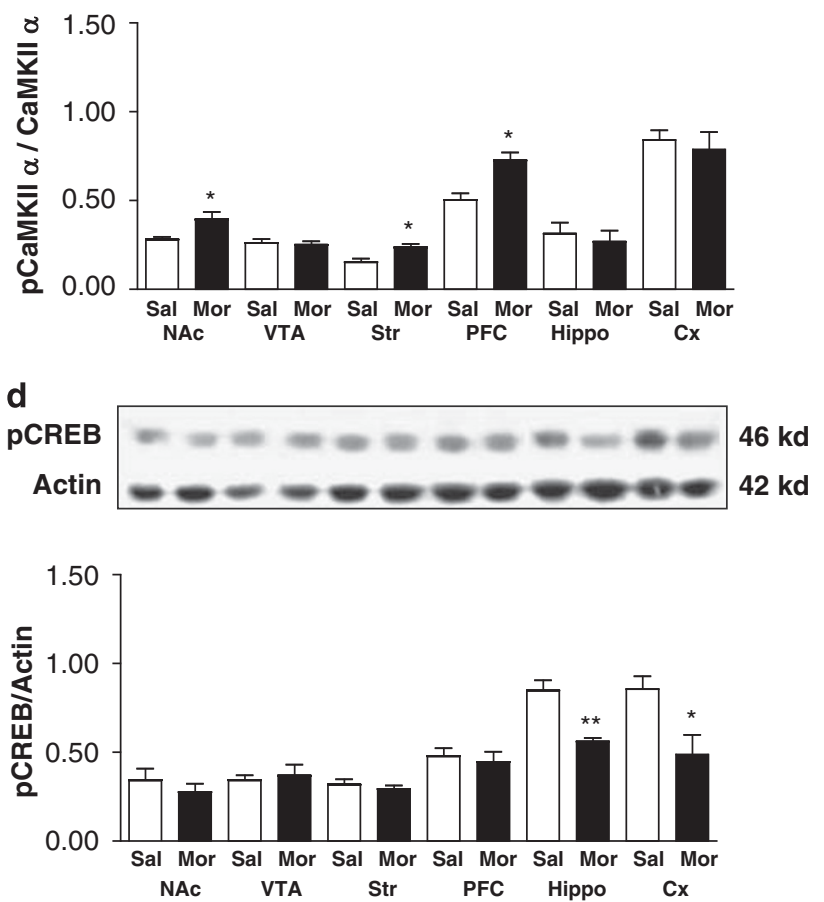

Figure 5 Phosphorylation of mitogen-activated protein kinase (MAPK), calcium/calmodulin-dependent kinase II (CaMKII), GluRI, and CREB in different brain regions of rats treated chronically with morphine without conditioned place preference (CPP). Rats were treated in their home cage with morphine or saline on days $\mid-5$ and on 8 and 9 as described in 'Materials and methods' section. Brain regions were dissected out and subjected to western blotting analysis using antibodies against pMAPK, total MAPK (a), pCaMKII, total CaMKII (b), pGluRI, total GluRI (c), pCREB, and actin (d) as described in 'Materials and methods' section. A representative western blot is shown for each of the proteins studied. Data from multiple experiments (4-6 animals per group) were tabulated and presented as relative ratio of phosphorylated/total forms (mean \pm SEM). One-way ANOVA with unpaired two-tailed $t$-test, $n=4-6$, * $p<0.05$, *** $p<0.01$.

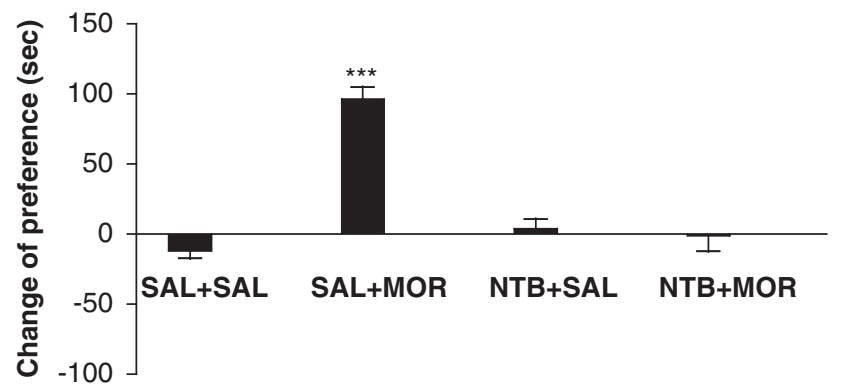

Figure 6 Effect of $\delta$-opioid receptor $(\delta \mathrm{OR})$ antagonist pretreatment on the sensitization to morphine conditioned place preference (CPP). Animals were pretreated with naltriben $(0.1 \mathrm{mg} / \mathrm{kg}$, s.c.) $15 \mathrm{~min}$ before the administration of morphine both during sensitization and conditioning as described in 'Materials and methods' section. Data represent the change of preference as described above (mean \pm SEM). One-way ANOVA with Tukey's test for multiple comparisons, $n=6-8$, ***** $p<0.00$ I.

\section{DISCUSSION}

In this study, we explored the effects of different paradigms of morphine administration on the phosphorylation levels of critical signaling molecules in different brain regions implicated in drug addiction. We observe increases in pCREB levels on expression of classical morphine CPP in the majority of the brain areas studied; this effect is also observed in response to the sensitization that develops to this conditioned behavior. In contrast, CREB phosphorylation is decreased on acute or repeated morphine administration (using a protocol comparable to the sensitization paradigm but that does not produce $\mathrm{CPP}$ ). These results are consistent with a critical role for CREB in the expression of morphinedependent conditioned behavior. Other studies have shown that CREB is induced in certain brain regions (including the hippocampus, Nac, and $\mathrm{PFC}$ ) in response to morphine $\mathrm{CPP}$, and that this regulation could change during acquisition, expression, or extinction of this conditioned behavior (Olson et al, 2005; Zhou and Zhu, 2006). The involvement of CREB in CPP is strengthened by studies showing that CREB knockout mice do not exhibit morphine-induced CPP (Walters and Blendy, 2001).

The transcriptional activity of CREB is regulated by the phosphorylation at Ser133 through various signaling pathways including MAPK, calcium, and stress signaling (Xing et al, 1998; Ribar et al, 2000). Therefore we also examined the modulation of MAPK and CaMKII phosphorylation under the different morphine treatment paradigms. We find increases in the levels of phosphorylated p44/42 MAPK in some brain regions on expression of morphine CPP (including those animals showing a sensitized response to morphine $\mathrm{CPP}$ ) but not under conditions of 

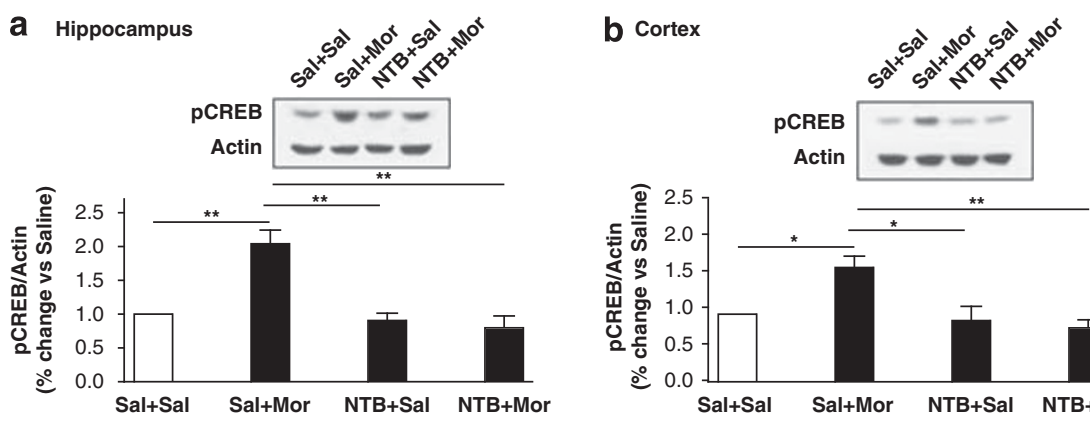

d NAC
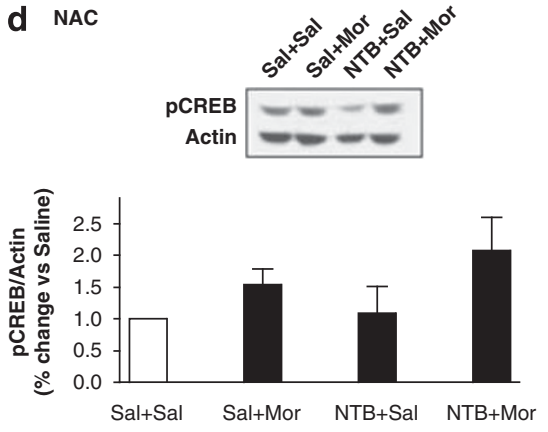

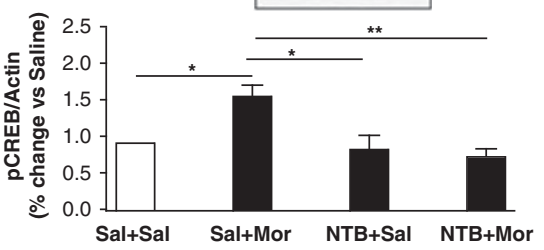

e VTA
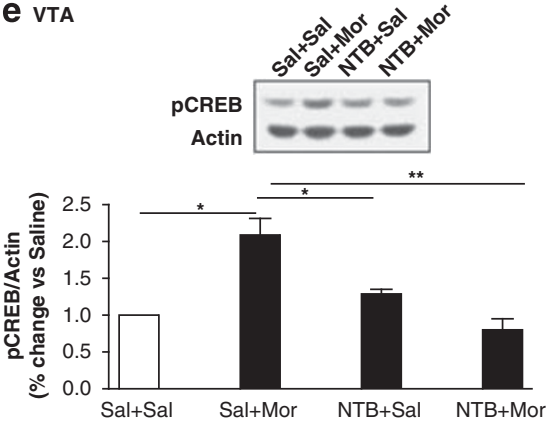

C PFC
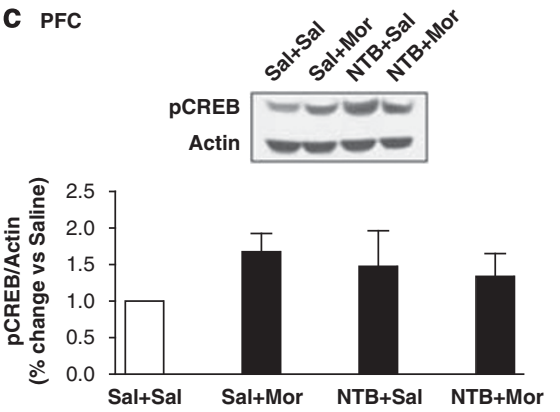

f Striatum
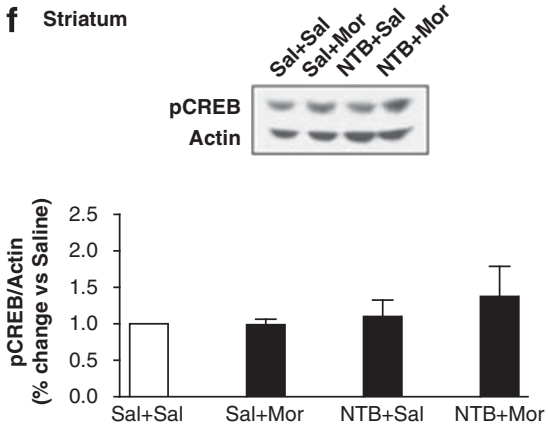

Figure 7 Effect of $\delta$-opioid receptor $(\delta \mathrm{OR})$ antagonist pretreatment on the induction of pCREB levels. The paradigm used for development of sensitization to morphine was modified in that animals were pretreated with naltriben $(0.1 \mathrm{mg} / \mathrm{kg}$, s.c.) 15 min before the administration of morphine both during sensitization and conditioning as described in 'Materials and methods' section. Brain regions were dissected out and subjected to western blotting analysis using antibodies against pCREB and actin as described in 'Materials and methods' section. A representative western blot is shown for each brain region examined. (a) Hippocampus, (b) Cortex, (c) PFC, (d) NAC, (e) VTA, (f) Striatum. Data from multiple experiments (6-8 animals per group) were tabulated and presented as relative ratio of phosphorylated/actin (mean \pm SEM). One-way ANOVA with Tukey's test for multiple comparisons, $n=6-8$, * $p<0.05, * * * 0.01$.
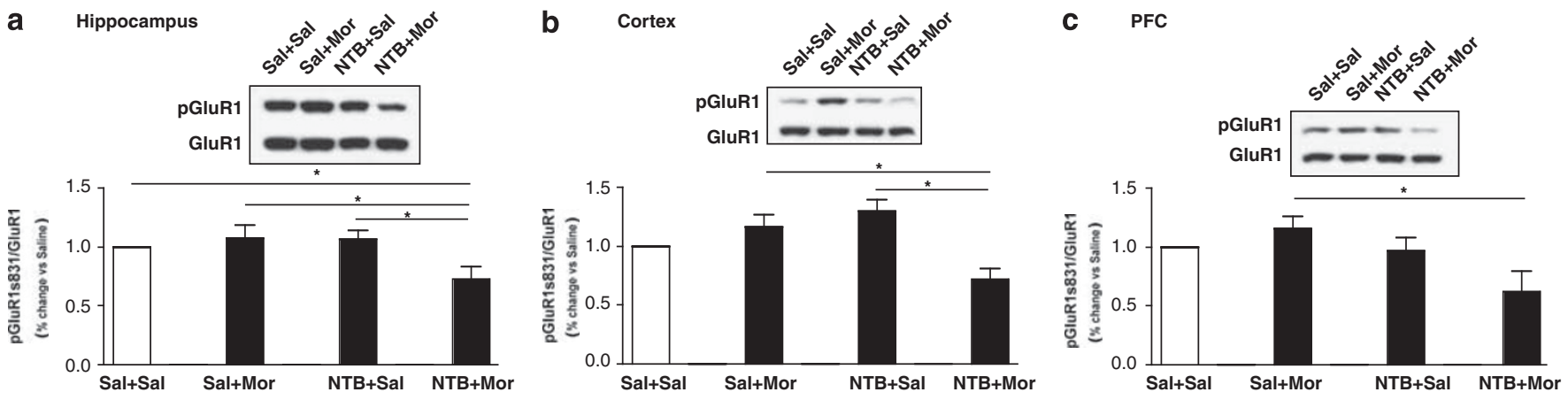

d NAC

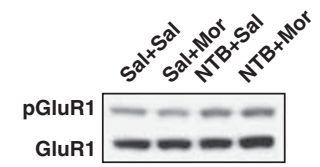

e VTA

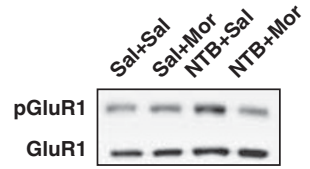

f Striatum
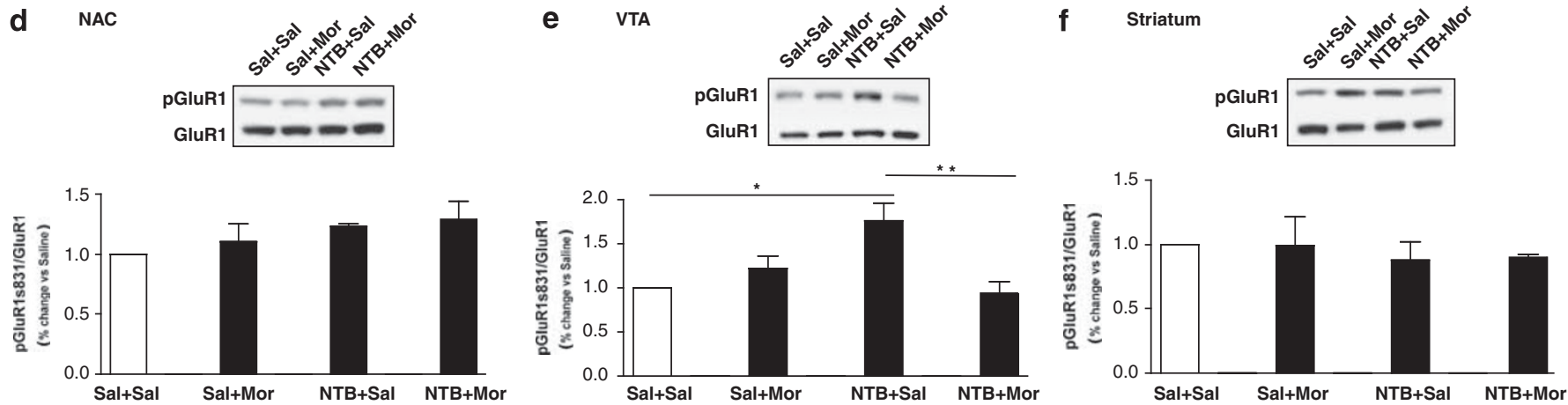

Figure 8 Effect of $\delta$-opioid receptor $(\delta \mathrm{OR})$ antagonist pretreatment on the induction of pGluRI levels. The paradigm used for development of sensitization to morphine was modified in that animals were pretreated with naltriben $(0.1 \mathrm{mg} / \mathrm{kg}, \mathrm{s} . \mathrm{c}$.) $15 \mathrm{~min}$ before the administration of morphine both during sensitization and conditioning as described in 'Materials and methods' section. Brain regions were dissected out and subjected to western blotting analysis using antibodies against phospho and total GluRI as described in 'Materials and methods' section. A representative western blot is shown for each brain region examined. (a) Hippocampus, (b) Cortex, (c) PFC, (d) NAC, (e) VTA, (f) Striatum. Data from multiple experiments (4-6 animals per group) were tabulated and presented as relative ratio of phosphorylated/total GluRI levels (mean \pm SEM). One-way ANOVA with Tukey's test for multiple comparisons, $n=6-8, * 0.05$, ** $p<0.01$. 
repeated morphine administration. This would indicate that phosphorylated MAPK could have a role in phosphorylating transcription factors and other molecules such as GluR1 during the expression of morphinedependent conditioned behavior. This is in contrast to CaMKII, which was found to be phosphorylated in select brain regions only under conditions of repeated morphine administration and morphine CPP but not under the sensitization that develops to this effect. It is likely that the increases in pCaMKII are directly related to morphine administration (and not to CPP). Further studies are needed to elucidate the role of CaMKII in the development of CPP to morphine in view of the fact that we do not observe significant changes in phosphorylated levels following sensitization to morphine CPP (that uses lower doses of morphine).

A recent study showed that morphine-induced CPP can be disrupted by locally inhibiting protein synthesis during conditioning in the hippocampus, amygdala, or NAc (Milekic et al, 2006). This disruption is long lasting, because morphine CPP is not reinstated by further conditioning. Therefore, the formation of contextual associations to morphine depends on the expression of proteins in certain brain regions. We, therefore, explored the effects of morphine administration on the expression of a candidate gene that may contribute to CREB action: the GluR1 subunit of the AMPA receptor. Our data show that the expression of morphine CPP induces GluR1 phosphorylation at Ser 831 in the hippocampus and cortex, brain regions involved in memory consolidation. In addition, sensitization to morphine CPP also leads to an increase in the phosphorylation of GluR1 in the hippocampus. Phosphorylation at Ser 831 has been shown to be mediated by protein kinase $\mathrm{C}$, which has been implicated in the induction and maintenance of long-term potentiation (Roche et al, 1996), an in vitro model of learning and memory. Therefore, by altering the phosphorylation state of GluR1, morphine could affect glutamatergic neurotransmission ( $\mathrm{Pu}$ et al, 2002; $\mathrm{Xu}$ et al, 2003), which would account for the synaptic plasticity observed during opiate addiction.

Research into the neurobiology of addiction and memory formation has focused largely on distinct, nonoverlapping brain circuits. The mesolimbic dopamine pathway, which includes dopamine-containing neurons in the VTA of the midbrain and their target areas in the limbic forebrain (Everitt and Wolf, 2002), has been extensively investigated because of its critical role in mediating the rewarding actions of drugs of abuse. Conversely, the medial temporal lobe, in particular the hippocampus, amygdala, and surrounding cortical areas, has been investigated because of its critical role in learning and memory (McGaugh, 2004). A few studies have implicated the hippocampus in mediating morphine CPP (Corrigall and Linseman, 1988; Ferbinteanu and McDonald, 2001). It is becoming increasingly clear that the neuronal circuits involved in addiction and memory largely overlap, and the PFC, NAc, VTA, hippocampus, and amygdala can be seen as highly interacting components of a larger circuit, which regulates different aspects of an individual's responses to environmental stimuli (Robbins and Everitt, 2002; Wolf, 2002). Our studies found that the expression of morphine CPP was associated with an increase in
CREB phosphorylation in the hippocampus and that this effect was also observed in the case of GluR1. In addition, the expression of morphine CPP correlated with an increase in PCREB in the VTA, supporting the notion that there is a significant overlap in the addiction and memory circuitry.

A major finding of this study is that administration of the $\delta \mathrm{OR}$ antagonist, naltriben, not only blocks the sensitized response to morphine CPP but also blocks the related increases in pCREB and pGluR1 levels in specific brain regions. Several studies have shown the involvement of $\delta \mathrm{OR}$ in mediating the rewarding effects of drugs of abuse through modification of dopamine neurotransmission in the mesolimbic pathway (Spanagel et al, 1990; Pentney and Gratton, 1991). In addition, it has been shown that repeated administration of $\delta \mathrm{OR}$ antagonist prevents sensitization to psychostimulants such as cocaine (Heidbreder et al, 1993). A previous report showed that repeated administration of the $\delta \mathrm{OR}$ antagonist, naltrindole, together with cocaine prevented the expression of cocaine CPP (Shippenberg and Heidbreder, 1995). Studies have also shown that administration of $\delta \mathrm{OR}$ ligands can modulate the behavioral effects of morphine (Porreca et al, 1992; He and Lee, 1998). Our data are also in agreement with a recent study that shows that administration of $\delta \mathrm{OR}$ antagonists disrupts sensitization of morphine CPP (Shippenberg et al, 2009), although the authors did not further explore the molecular mechanisms underlying these effects. This $\delta \mathrm{OR}-$ antagonist-induced modulation of the sensitization to morphine CPP is likely due to the physical association between $\mu \mathrm{OR}$ and $\delta \mathrm{OR}$ (Gomes et al, 2004). Indeed, Gomes et al (2004) have shown that treatment with $\delta$ OR ligands alters the number of $\mu \mathrm{OR}$ binding sites, thereby affecting the potency of morphine. Thus, by targeting $\delta$ OR one could develop therapies for the prevention and treatment of morphine addiction.

In summary, we have carried out a systematic study to examine the effects of morphine administration on phosphorylation states of opiate-mediated signaling molecules. As a result, we observed that expression of morphine $\mathrm{CPP}$ as well as the sensitization to this conditioned response correlate with an increase in pCREB and pGluR1 levels in brain areas such as the hippocampus, cortex, and VTA. Interestingly, the $\delta \mathrm{OR}$ antagonist blocks both the sensitized response to morphine CPP and its related increases in phosphorylation of CREB and GluR1. The results of this study would help in the identification of the mechanisms that underlie the behavioral adaptations in response to drug-associated environmental cues.

\section{ACKNOWLEDGEMENTS}

We thank members of C Alberini's lab for help with the behavioral assays. We also thank Dr Toni Shippenberg for advice. This work was supported in part by NIH-NIDA Grants DA008863 and DA019521 (to LAD).

\section{DISCLOSURE}

The authors declare no conflict of interest. None of the authors have received compensation for professional 
services in any of the previous three years, or anticipate receiving such compensation in the near future.

\section{REFERENCES}

Abdelhamid E, Sultana M, Portoghese P, Takemori A (1991). Selective blockage of delta opioid receptors prevents the development of morphine tolerance and dependence in mice. J Pharmacol Exp Ther 258: 299-303.

Bardo MT, Rowlett JK, Harris MJ (1995). Conditioned place preference using opiate and stimulant drugs: a meta-analysis. Neurosci Biobehav Rev 19: 39-51.

Carlezon Jr WA, Duman RS, Nestler EJ (2005). The many faces of CREB. Trends Neurosci 28: 436-445.

Corrigall WA, Linseman MA (1988). Conditioned place preference produced by intra-hippocampal morphine. Pharmacol Biochem Behav 30: 787-789.

Daniels D, Lenard N, Etienne C, Law P, Roerig S, Portoghese P (2005). Opioid-induced tolerance and dependence in mice is modulated by the distance between pharmacophores in a bivalent ligand series. Proc Natl Acad Sci USA 102: 19208-19213.

Everitt BJ, Wolf ME (2002). Psychomotor stimulant addiction: a neural systems perspective. J Neurosci 22: 3312-3320.

Ferbinteanu J, McDonald RJ (2001). Dorsal/ventral hippocampus, fornix, and conditioned place preference. Hippocampus 11: 187-200.

Fuchs RA, Evans KA, Ledford CC, Parker MP, Case JM, Mehta RH et al (2005). The role of the dorsomedial prefrontal cortex, basolateral amygdala, and dorsal hippocampus in contextual reinstatement of cocaine seeking in rats. Neuropsychopharmacology 30: 296-309.

Fukuda K, Kato S, Morikawa H, Shoda T, Mori K (1996). Functional coupling of the delta-, mu-, and kappa-opioid receptors to mitogen-activated protein kinase and arachidonate release in Chinese hamster ovary cells. J Neurochem 67: 1309-1316.

Gomes I, Gupta A, Filipovska J, Szeto HH, Pintar JE, Devi LA (2004). A role for heterodimerization of mu and delta opiate receptors in enhancing morphine analgesia. Proc Natl Acad Sci USA 101: 5135-5139.

He L, Lee NM (1998). Delta opioid receptor enhancement of $\mathrm{mu}$ opioid receptor-induced antinociception in spinal cord. J Pharmacol Exp Ther 285: 1181-1186.

Heidbreder C, Goldberg SR, Shippenberg TS (1993). Inhibition of cocaine-induced sensitization by the delta-opioid receptor antagonist naltrindole. Eur J Pharmacol 243: 123-127.

Hyman SE, Malenka RC (2001). Addiction and the brain: the neurobiology of compulsion and its persistence. Nat Rev Neurosci 2: 695-703.

Kalivas PW, Stewart J (1991). Dopamine transmission in the initiation and expression of drug- and stress-induced sensitization of motor activity. Brain Res Brain Res Rev 16: 223-244.

Koob GF, Sanna PP, Bloom FE (1998). Neuroscience of addiction. Neuron 21: 467-476.

Law PY, Wong YH, Loh HH (2000). Molecular mechanisms and regulation of opioid receptor signaling. Annu Rev Pharmacol Toxicol 40: 389-430.

McGaugh JL (2004). The amygdala modulates the consolidation of memories of emotionally arousing experiences. Annu Rev Neurosci 27: 1-28.

Milekic MH, Brown SD, Castellini C, Alberini CM (2006). Persistent disruption of an established morphine conditioned place preference. J Neurosci 26: 3010-3020.

Morris RG, Moser EI, Riedel G, Martin SJ, Sandin J, Day M et al (2003). Elements of a neurobiological theory of the hippocampus: the role of activity-dependent synaptic plasticity in memory. Philos Trans R Soc London B Biol Sci 358: 773-786.
Mueller D, Perdikaris D, Stewart J (2002). Persistence and druginduced reinstatement of a morphine-induced conditioned place preference. Behav Brain Res 136: 389-397.

Narita M, Funada M, Suzuki T (2001). Regulations of opioid dependence by opioid receptor types. Pharmacol Ther 89: 1-15.

Nestler EJ (1997). Molecular mechanisms of opiate and cocaine addiction. Curr Opin Neurobiol 7: 713-719.

Nestler EJ (2001). Molecular neurobiology of addiction. Am J Addict 10: 201-217.

O'Brien CP, Childress AR, McLellan AT, Ehrman R (1992). Classical conditioning in drug-dependent humans. Ann NY Acad Sci 654: 400-415.

Olson VG, Zabetian CP, Bolanos CA, Edwards S, Barrot M, Eisch AJ et al (2005). Regulation of drug reward by cAMP response element-binding protein: evidence for two functionally distinct subregions of the ventral tegmental area. J Neurosci 25: 5553-5562.

Pentney RJ, Gratton A (1991). Effects of local delta and mu opioid receptor activation on basal and stimulated dopamine release in striatum and nucleus accumbens of rat: an in vivo electrochemical study. Neuroscience 45: 95-102.

Polakiewicz RD, Schieferl SM, Gingras AC, Sonenberg N, Comb MJ (1998). mu-Opioid receptor activates signaling pathways implicated in cell survival and translational control. J Biol Chem 273: 23534-23541.

Porreca F, Takemori AE, Sultana M, Portoghese PS, Bowen WD, Mosberg HI (1992). Modulation of mu-mediated antinociception in the mouse involves opioid delta-2 receptors. J Pharmacol Exp Ther 263: 147-152.

Portoghese P, Sultana M, Nagase H, Takemori A (1992). A highly selective delta-1-opioid receptor antagonist: 7-benzylidenenaltrexone. Eur J Pharmacol 218: 195-196.

$\mathrm{Pu}$ L, Bao GB, Xu NJ, Ma L, Pei G (2002). Hippocampal longterm potentiation is reduced by chronic opiate treatment and can be restored by re-exposure to opiates. J Neurosci 22: 1914-1921.

Ribar TJ, Rodriguiz RM, Khiroug L, Wetsel WC, Augustine GJ, Means AR (2000). erebellar defects in $\mathrm{Ca}^{2+} /$ calmodulin kinase IV-deficient mice. J Neurosci 20: RC107.

Robbins TW, Everitt BJ (2002). Limbic-striatal memory systems and drug addiction. Neurobiol Learn Mem 78: 625-636.

Roche KW, O'Brien RJ, Mammen AL, Bernhardt J, Huganir RL (1996). Characterization of multiple phosphorylation sites on the AMPA receptor GluR1 subunit. Neuron 16: 1179-1188.

Sanchez-Blazquez P, Garcia-espana A, Garzon J (1997). Antisense oligodeoxynucleotides to opioid mu and delta receptors reduced morphine dependence in mice: role of delta-2 opioid receptors. J Pharmacol Exp Ther 280: 1423-1431.

Shippenberg TS, Chefer VI, Thompson AC (2009). Delta-opioid receptor antagonists prevent sensitization to the conditioned rewarding effects of morphine. Biol Psychiatry 65: 169-174.

Shippenberg TS, Heidbreder C (1995). The delta-opioid receptor antagonist naltrindole prevents sensitization to the conditioned rewarding effects of cocaine. Eur J Pharmacol 280: 55-61.

Shippenberg TS, Heidbreder C, Lefevour A (1996). Sensitization to the conditioned rewarding effects of morphine: pharmacology and temporal characteristics. Eur J Pharmacol 299: 33-39.

Shippenberg TS, Lefevour A, Thompson AC (1998). Sensitization to the conditioned rewarding effects of morphine and cocaine: differential effects of kappa-opioid receptor agonist U69593. Eur J Pharmacol 354: 27-34.

Sofouglu M, Portoghese P, Takemori A (1991). Differential antagonism of delta opioid agonists by naltrindole and its benzofuran analog (NTB) in mice: evidence for delta opioid receptor subtypes. J Pharmacol Exp Ther 257: 676-680.

Spanagel R, Herz A, Shippenberg TS (1990). The effects of opioid peptides on dopamine release in the nucleus accumbens: an in vivo microdialysis study. J Neurochem 55: 1734-1740. 
Walters CL, Blendy JA (2001). Different requirements for cAMP response element binding protein in positive and negative reinforcing properties of drugs of abuse. J Neurosci 21: 9438-9444.

Wolf ME (2002). Addiction: making the connection between behavioral changes and neuronal plasticity in specific pathways. Mol Interv 2: 146-157.

Xing J, Kornhauser JM, Xia Z, Thiele EA, Greenberg ME (1998). Nerve growth factor activates extracellular signal-regulated kinase and p38 mitogen-activated protein kinase pathways to stimulate CREB serine 133 phosphorylation. Mol Cell Biol 18: $1946-1955$.
Xu NJ, Bao L, Fan HP, Bao GB, Pu L, Lu YJ et al (2003). Morphine withdrawal increases glutamate uptake and surface expression of glutamate transporter GLT1 at hippocampal synapses. J Neurosci 23: 4775-4784.

Zhou LF, Zhu YP (2006). Changes of CREB in rat hippocampus, prefrontal cortex and nucleus accumbens during three phases of morphine induced conditioned place preference in rats. J Zhejiang Univ Sci B 7: 107-113.

Zhu Y, King M, Schuller A, Nitsche J, Reidl M, Elde R et al (1999). Retention of supraspinal delta-like analgesia and loss of morphine tolerance in delta opioid receptor knockout mice. Neuron 24: 243-252. 